\title{
Disaster Series: Disaster Planning for Perinatal/Neonatal Medicine: Rethinking the Process and Caring for Mothers. Infants and Technology Dependent Infants - The Time is NOW
}

Daved van Stralen, M.D., Elba Fayard, M.D., Julia Paz, D.O., Louisa Shelby RN, BSN, IBCLC, Mitchell Goldstein, M.D., MBA, T. Allen Merritt, M.D, MHA

\section{Introduction:}

Disasters that affect health care delivery seem to be happening with increased frequency and can be found all around us. Within the last month, the Caldor Fire in Northeast California resulted in the evacuation of Barton Memorial Hospital in South Lake Tahoe (Sacramento Bee, August 30, 2021). Hurricane Ida caused the loss of electrical power and loss of water to New Orleans Children's Hospital, making an evacuation of their NICU and other patients to other hospitals necessary, according to the Chief Medical Officer, Dr. Mark Kline on CBS Good Morning America (August $30 / 2021$ ). We aim to review how disasters, both natural and manmade, threaten the well-being, indeed the very lives of pregnant women, newborns, and especially the lives of technology-dependent infants and children. We offer novel approaches for critical decisions and actions that accomplish our life-saving goals.

\section{"We aim to review how disasters, both natural and man-made, threaten the well- being, indeed the very lives of pregnant women, newborns, and especially the lives of technology-dependent infants and children. We offer novel approaches for critical decisions and actions that accomplish our life-saving goals."}

Disasters may be very sudden and unexpected. Planning care delivery by highly skilled specialized caregivers, compromised in their routine functions by unanticipated damage to physical facilities, loss of electrical power, or inability of scheduled caregivers to work as designated due to environmental disruption and travel hazards is of utmost importance. Health care workers may also suffer unexpected injury or trauma or even widespread and progressive illnesses. We offer an action plan for emergency management officials and administrators, obstetric and pediatric

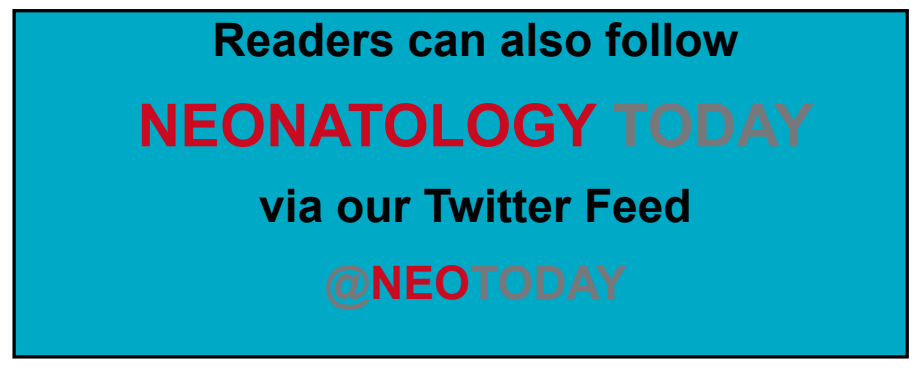

providers, and ancillary health care professionals, responding to emergent and disruptive challenges requiring flexible actions to protect patients and healthcare workers.

Emergency Management Services in many States are defined by statute. For example, in Oregon, "Emergency" means a human-created or natural event or circumstance that causes or threatens the widespread loss of life, injury to person or property, human suffering, or financial loss, including but not limited to:

a) Fire, explosion, flood, severe weather, landslides or mudslides, drought, earthquake, volcanic activity, tsunamis, or other oceanic phenomena, spills or releases of oil or hazardous material as defined, contamination, utility or transportation emergencies, disease, blight, infestation, civil disturbance, riot, sabotage, acts of terrorism and war

b) A rapid influx of individuals from outside the states, rapid migration of individuals from one part to another, or a rapid displacement of individuals in the influx, migration or displacement results from the type of event or circumstance described in paragraph (a) of this subsection (Oregon Revised Statutes 401.025 Chapter 401, June 2018). State Emergency Management and Services do not fully cover maternal, perinatal care, and maternal/infant aftercare, including intensive care services, either continuation or initiation, or essential services required by technology-dependent children. The response from the Office of Emergency Management Services, often including a proclamation by the Governor, takes time. This time is best described as a "fallacy of concreteness" when those on the ground, at or near the scene, and receiving casualties and evacuees will act on their own to make critical decisions described as "learn by doing" with reciprocal feedback (1). A disaster often constrains available information from outside for the immediate access location of support personnel. Though they always act in a way that makes sense to them, available information and guidance may be limited, imperfect, and changing, thus leading to gaps between system plans and operator practice. There will also be gaps between discrete concepts and their continuous perceptions of rapidly changing situations. Rasmussen described the purpose of operators and responders in complex technological systems. they are flexible, can learn to adapt to the system's peculiarities, and thus are expected to plug the holes in the designer's imagination. (2).

In Oregon, the Oregon Health Authority, among other collaborating state agencies, has primary responsibility for emergency medical responses. Emergency Support Functions (ESFs) for pregnant women, infants, and children fall under two major categories. ESF-6 Mass Care and ESF-8 for Health and Medical Services. Pregnant women, lactation support, women with newborns, infants on CPAP, feeding tubes, or gastrostomy would be triaged to a Mass Care unit. High-risk pregnancies, women in labor, women with newborns with medical complications such as those on high flow nasal cannula would be triaged to Health and Medical Units (Tables I and II). The California Hospital Association has created the "Hospital Activation of the Emergency Operations Plan Checklist" among other checklists for unique situations such as "active 
shooter," Emergency food supplies for hospitals, and hospital water disruption. (3) (CHA Hospital Preparedness Program (https:// www.calhospitalprepare.org/cha-tools, accessed 8/4/21).

\section{"However, the California Emergency Services Act is short on how this duty applies to pregnant women, newborn infants, or technology-dependent infants and children."}

The California Emergency Services Act (8551) states that "the state has long recognized its responsibility to mitigate the effects of natural, man-made, or war-caused emergencies which result in conditions of disaster or extreme peril to life and property; and the resources of the state to protect the health and safety and preserve the lives and property of the people of the state." It outlines how the Governor and various chief executives and governing bodies of political subdivisions of California have emergency powers to provide for state-based assistance and provides for a state agency "Office of Emergency Services" to prescribe the powers and duties of the director of that office. However, the California Emergency Services Act is short on how this duty applies to pregnant women, newborn infants, or technology-dependent infants and children.

Kaiser Permanente in California has adopted a Hazard and Vulnerability Assessment tool for Human Related events. It allows risk calculation over an array of hospital resources and functions, of which preparedness, internal response, and external response are critical factors for these calculations. The human impact, property, and business impact are also calculated for risk. This system augmented their response to wildfires affecting Kaiser beneficiaries and facilities, some of which were severely affected in Santa Rosa, California, in 2017 (4) by developing a turnkey command center that opens a command center before the threat becomes acute, identifies interdependencies, and activate resources, considering incremental action as needed. They emphasize "the reality is that the risk our facilities face today are different than what we imagined and faced a decade ago-and that a rapidly changing climate poses yet unforeseeable hazards for the future." (5).

Applicable definitions in disaster preparation, response, and recovery include (6)

1. All-Hazards: An approach for prevention, protection, preparedness, response, and recovery that addresses a full range of threats and hazards, including domestic terrorist attacks, natural and manufactured disasters, accidental disruptions, and other emergencies.

2. Assisting Agency: An agency or organization providing personnel, services, or other resources to the agency with direct responsibility for incident management.

3. Cache: A pre-determined complement of tools, equipment, and/or supplies stored in a designated location, available for incident use.

4. Emergency Operations Plan: An ongoing plan for responding to a wide variety of potential hazards.
5. Evacuation: The organized, phased, and supervised withdrawal, dispersal, or removal of patients/personnel from dangerous or potentially dangerous areas and their reception and care in safe areas.

6. Incident: An occurrence, natural or man-made, for which an emergency response protects life or property. Some examples of incidents include, but are not limited to, earthquakes, hurricanes, tornadoes, tsunamis, wildfires or urban fires, floods, nuclear accidents, hazardous materials spills, aircraft accidents, war-related disasters, terrorist attacks, civil unrests, and public health emergencies.

7. Mitigation: Mitigation is the effort to reduce loss of life and property by lessening the impact of disasters. Mitigation is taking actions ahead of time to prevent or minimize human safety and property losses. These actions reduce or eliminate the need for emergency response and significantly reduce the recovery period.

8. National Incident Management System: The National incident Management System (NIMS) identifies concepts and principles that guide emergencies from preparedness to recovery. The NIMS provides a consistent, nationwide approach and vocabulary for multiple governmental, private, and jurisdictions to work together in response efforts, regardless of cause. This guidance ensures effective and integrated preparedness, planning, and response and reduces loss of life or property and harm to ecosystems.

9. Preparedness: A continuous cycle of planning, organizing, training, equipping, exercising, evaluating, and taking corrective action to ensure effective coordination during incident response.

10. Prevention: Actions to avoid an incident or to intervene to stop an incident from occurring. Prevention involves actions to protect lives and property. It consists in applying intelligence and other information to a range of or quarantine and, as appropriate, specific law enforcement operations aimed at deterring, preempting, interdicting, or disrupting illegal activity and apprehending potential perpetrators.

11. Resources: Personnel and significant items of equipment, supplies, and facilities available for potentially available for assignment to incident operations and for which status is maintained. Various types of equipment for maternal, perinatal, neonatal, and technology-dependent children has also been endorsed by the California Department of Public Health and the Disaster Preparedness Task Force of the Naval Medical Center, San Diego. (7)

\section{In 2015 the California Perinatal Quality Care Collaborative and Loma Linda University Children's Hospital prepared a toolkit for NICU disaster preparedness and described steps to prepared needed equipment and personnel from multiple disciplines and execute plans if a disaster occurred. (8)"}


In 2015 the California Perinatal Quality Care Collaborative and Loma Linda University Children's Hospital prepared a toolkit for NICU disaster preparedness and described steps to prepared needed equipment and personnel from multiple disciplines and execute plans if a disaster occurred. (8) However, Eskandar-Afshari et al. reported a survey of 84 NICUs in California in 2018 (8). They found that $10 \%$ did not participate in annual drills, $44 \%$ did once a year, $36 \%$ did twice per year, and $10 \%$ did more than twice per year. They concluded that NICU had systems in place and plans for various disasters. Still, there was inconsistent participation in hospital training for NICU disaster preparedness and a lack of sufficient exercises for unit readiness if a disaster were to occur $(9,10)$. Barfield et al. (11) prepared a detailed clinical report for "Disaster Preparedness in Neonatal Intensive Care Units" in 2017. Still, in the subsequent four years, national emergencies such as the COVID-19 pandemic, regional wildfires burning entire communities such as Santa Rosa, California, and its hospital, or tornados devastating a St John's Medical Center in Joplin, Missouri, airborne toxins from burning chemical fires at refineries, and unprecedented heat attributed to climate change with temperatures up to 130 degrees $F$, have disrupted healthcare services to pregnant women, their newborn infants, neonatal intensive care units, and transport systems. These function well in the absence of these disasters. Obstetricians, neonatologists, pediatricians and supporting perinatal nurses, lactation support, respiratory care practitioners, pharmacists, social workers, and others working within regionalized perinatal care delivery systems must collaborate and plan a collective response when a disaster strikes. The perinatal team shares an obligation to create policies to optimize care provision to mothers, infants, and especially technology-dependent infants, such as those in an NICU, focusing on these vulnerable patients by providing trauma-informed care. Furthermore, triage and delegation of resources may be limited over extended periods, and the lack of resources creates ethical issues that are best anticipated and presented for their advanced approval.

"In 2013 the American Academy of
Pediatrics, with support from the
Centers for Disease Control and
Prevention (Pediatric Preparedness
Resource Kit) (12), focused on the
H1NI pandemic, provided a pediatric
toolkit regarding policies concerning
pediatricians and children in disasters.
Still, in the decade since, there has been
a paucity of a creative and thoughtful
redesign of systems of care or function
during the multitude of tragedies that
have affected mothers, infants, and
technological dependent infants in NICU
settings."

In 2013 the American Academy of Pediatrics, with support from the Centers for Disease Control and Prevention (Pediatric Preparedness Resource Kit) (12), focused on the H1NI pandemic, provided a pediatric toolkit regarding policies concerning pediatricians and children in disasters. Still, in the decade since, there has been a paucity of a creative and thoughtful redesign of systems of care or function during the multitude of tragedies that have affected mothers, infants, and technological dependent infants in NICU settings. Pregnant women, women in labor, newborns, and infants in NICUs are particularly vulnerable. Because of the dynamic changes in a pregnant woman's physiology, emotional distress may precipitate premature labor. Infants, because of their small size, physiologic immaturity, and baseline requirement for technology or supplemental oxygen, specialized nutritional needs, medication administration, and monitoring of cardiorespiratory function, as well as diagnostic information gathering and life-sustaining support, can be imperiled. Disasters may disrupt a mother's or infant's protective surroundings (warmth, light, humidity, oxygen, and air mixtures, or filtered air, as well as diagnostic and therapeutic resources. Different types of disasters may mandate immediate evacuation to a safe place and disrupt the expected medical care by a previously established professional relationship. Disasters may lead to uncontrolled physiologic changes when supplies to supplemental oxygen are suddenly disrupted. Indeed, "ideal" physiology, such a maintaining an "ideal" $\mathrm{SaO}_{2}$ of $92-95 \%$, may need to be substituted for lower saturations in room air that are still survivable.

Disasters have many different origins. Natural and man-made disasters (and even hybrids) exist, such as the reactor meltdown in Fukushima, Japan, following the damage from a tsunami at the Dai-ichi Nuclear Power Station (a nuclear reactor closer to the epicenter was undamaged from the same tsunami). Chemical releases caused by natural hazard events and disasters. Recent fires at Baytown and Harris County Texas Exxon Mobil refinery in 2019 injured 37 and required a "shelter in place" order for residents as ammonia, benzene, and propylene were released into the atmosphere, endangering thousands. The Galveston Bay Marathon Oil refinery fire resulted in the release of hydrogen fluoride- a highly toxic chemical. More recently, biological disasters such as anthrax, Zika, HiN1 influenza, and the ongoing COVID-19 (and variants) pandemic or whether from terrorist activities or animal to human and human to human page 4contact have resulted in national or worldwide calamities. Responding to each type of disaster has unique elements depending on the risks and threats imposed on pregnant women, those in labor, post-partum women, and their infants, especially infants and children dependent on medical technologies.

\section{Increased risk for Mothers During Disasters:}

One in five women of childbearing age is pregnant in a disaster setting. The obstetric care needs of these women are constant during the various stages of disaster response and recovery (13); multiple studies have documented an association between disasters and their impact on pregnancies. Early pregnancy loss, increased birth defects (possibly related to toxic exposures, smoke exposure, pandemic infections such as Zika or COVID-19) or lack of preventive measures with inadequate nutrition, folic acid, and iron supplements contribute to placental abruption, preterm birth, and low birth weight infants (14). Pregnant women are at increased risk of maternal death because of limited or reduced access to prenatal care, assisted delivery, emergency obstetric care, and anesthesia. Increased rates of peripartum infections have 
also been reported (15). In a systematic review of health outcomes after floods, mortality rates in general increased up to 50 percent in the first year after the event. Many of the deaths were associated with increased exposure to infectious agents (16) in this study. Rates of spontaneous abortion and birth defects have been documented following disasters, contributing to decreased fecundity, presumably secondary to traumatic or toxic exposures. In a prospective study of 301 pregnant women who experienced Hurricane Katrina, preterm birth increased threefold with preterm birth rates of 14 percent versus 5 percent in those not experiencing such a disaster. (17) There was no significant difference in preterm birth for women without post-traumatic stress disorder or depression. While the etiologies of poorer pregnancy outcomes are unclear, the intensity of the disaster experience appears to be a risk factor (18). In a study that compared the outcomes of women and infants before and after the 1997 Red River flood in North Dakota, there was an increase in underlying maternal medical morbidities (anemia, lung disease, uterine bleeding), low birth weight, and preterm delivery following the event. (19).

\section{"In a study that compared the outcomes of women and infants before and after the 1997 Red River flood in North Dakota, there was an increase in underlying maternal medical morbidities (anemia, lung disease, uterine bleeding), low birth weight, and preterm delivery following the event. (19)."}

\section{Action Planning: Caring for Mothers During Disasters:}

Both basic and comprehensive emergency obstetric and newborn care services need to be considered in disaster planning and established as soon as possible, both during and following a disaster. The creation of a minimum initial service package (MISP) for managing reproductive health services helps disaster planners and field workers anticipate the reproductive health needs of women within a population. Operational systems for both treating and triaging, working simultaneously with each guiding the other, and using joint decision-making are critical. Knowledge of sitespecific data on crude birthrates, contraceptive prevalence rate, sexually transmitted infection prevalence rates, and maternal mortality ratio can be computer to estimate:

- $\quad$ Number of currently pregnant women

- $\quad$ Number of anticipated births

- Complications

\section{Cesarean deliveries and Neonatal Resuscitation:}

This information can be used to estimate needs when preparing for obstetric care and for stockpiling supplies for use during a disaster. The use of the MISP calculator helps determine the estimated number of pregnant women, the anticipated number of women who will deliver or miscarry, and the permit ordering of obstetric and newborn supplies. An Obstetrics and Gynecology specialist should take a leadership role in assessing the availability and creation of a robust supply chain and proper replacement of equipment, medications, and supplies for potential deliveries and neonatal care. As transportation to existing obstetric facilities may be significantly hampered, having onsite medications and delivery kits, and neonatal care provisions are necessary. Medications deemed essential for deliveries include oxytocin, misoprostol, and tranexamic acid. Also, neonatal care warmers, epinephrine, normal saline, air, and oxygen with pressure regulators for bag and mask ventilation or $\mathrm{T}$ piece resuscitation devices are essential. Because clean water and soap may be less available, using alcohol-based cleanser solutions for handwashing and site preparation is invaluable. Ideally, a portable ultrasound device will be available for fetal and neonatal imaging.

\section{Impact of Disaster Setting on Pregnancy and Delivery Care}

Disaster causes an acute resource scarcity; thus, healthcare workers must consider the availability of resources to safely facilitate procedural and postprocedural care before evacuation to a higher level of care. For instance, the indications for cesarean delivery in an evacuation center or even a field hospital have increased risk for infection, blood loss, and a more limited capacity to address complications. Clinicians must prioritize procedures for women requiring immediate life-saving interventions, followed by fetal intervention and immediate newborn care. For example, performing routine cesarean delivery for a breech presentation may be deferred in favor of cesarean deliveries only for women with abdominal trauma. The procedure will aid maternal resuscitation. Potential contingencies and decision rules such be discussed in the planning stage and among the assembled team of healthcare providers. In a qualitative interview of 11 women who were pregnant during Hurricane Katrina in New Orleans, the hurricane disrupted pregnancy plans and led to great anxiety and uncertainty. Although none received direct injuries, three underwent cesarean delivery as indicated by usual obstetric criteria in a resource-limited environment.

\section{Perinatal Triage and Response Considerations:}

Disaster triage is the most important and psychologically the most challenging part of the medical response in significant disasters. Disaster triage aims to do the greatest good for the greatest number of patients with the determinants based on the severity of injury/disease, the likelihood of survival, and available resources. Disaster medical triage may be conducted at three (or more) different levels. Field triage in acute (non-ambulatory) versus nonacute (ambulatory) settings such as an evacuation center or by Emergency Medical services must occur. Medical triage involves a rapid assessment of women to identify life-threatening injuries, determine those with the best chance of survival, and allocate care in settings of insufficient resources. If possible, laboring women should be referred to an appropriate health care facility for delivery. Before the transfer, the clinician should assess, as best as possible, the gestation age of the patient, available levels of neonatal intensive care, and the limits of fetal viability in a specific disaster setting. The types of facilities are typically divided into Basic Emergency Obstetric and Newborn Care (BEmONC) and Comprehensive Emergency Obstetric and Newborn Care units (CEmONC) (16). BEmONC provided 24 -hour access to skilled birth attendants and possible access to intramuscular and/or intravenous antibiotics, uterotonics, and anticonvulsants. Professional services may include manual extraction of the placenta, uterine 
evacuation in cases of a retained placenta following delivery or miscarriage, assisted vacuum delivery, and neonatal resuscitation using NRP. Women candidates for BEmONC delivery include primiparous, have a history of intrauterine fetal death, hemorrhage, or assisted vaginal delivery (vacuum or forceps, or grand multiparity).

CEmONC facilities typically serve as regional referral medical centers that provide all services described above but have facilities to perform cesarean delivery and hysterectomy, offer blood transfusions, and provide neonatal intensive care for premature and low birth weight infants. Women who benefit from delivering at the CEmONC facility include those who require cesarean delivery or those in whom cesarean delivery is likely.

\section{"Post-partum care is provided within the first 76 hours that follow the delivery. Disasters are often accompanied by injuries and illnesses that should be treated within the context of post-partum care in affected women. Knowledge of normal post-partum physiology, the basics of post-partum care, and the potential risks during this time can assist in determining when there is a need for intervention."}

\section{Postpartum Woman:}

Post-partum care is provided within the first 76 hours that follow the delivery. Disasters are often accompanied by injuries and illnesses that should be treated within the context of post-partum care in affected women. Knowledge of normal post-partum physiology, the basics of post-partum care, and the potential risks during this time can assist in determining when there is a need for intervention. Both mothers and their newborns are vulnerable during the post-partum days. An estimated $65 \%$ of all maternal deaths occur after the delivery, and almost half of these postpartum deaths occur within 24 hours of delivery (21). Maternal bleeding and hypertensive disorders can occur after birth and up until six weeks post-partum. The first 24 hours of a newborn infant's life are critical. The maternal-infant dyad must be carefully monitored for breastfeeding, maintaining infant temperature, and urination and stooling patterns. Two-thirds of infant death occurs within the first week after birth, and $50 \%$ occur within the first 24 hours after birth (22).

\section{Maintaining the Readiness of the Obstetric Team}

Physicians and other birth attendants interested in providing obstetric care during a disaster should obtain training for obstetric management during disasters so that they will continue to carry out their professional responsibilities in austere environments. The following attributes and traits of staff are helpful.

\section{Attributes that Facilitate Birthing Center staff coordination}

\section{and functioning during a disaster}

1. Able to work in hardship positions

2. Are multifunctional

3. Integrate well into a camp or military-like environment

4. Able to make complex and difficult decisions

5. Able to work cooperatively within a hierarchical structure of the organizational response.

6. Able to coordinate between Obstetric and Pediatric providers and local public health authorities and disaster or incident commands.

Leeb and coworkers report a framework for coordination between obstetric and pediatric provides in their combined response to the Zika outbreak in the U.S from 2015 to 2017 (23). They provide a framework to help coordinate the care of pregnant women and their infants exposed to congenital threats. Their framework identifies critical information to inform care, establish health care touchpoints and communication/infants transfer pathways. They created the "children's Interdisciplinary Care page Coordination Framework based on a frame that (1) identifies and collects critical information to inform care. These are primary pathways of communication and modes of transfer, essential health care touchpoints for the maternal-infant dyad, and health care providers to transmit critical information to other health care providers, the patient, and family. They provide strategies to facilitate implementation during a public health crisis. In their case, it was applied to the Zika epidemic.

1. Strategy 1 Identifying critical information during an emergency response to a novel biologic agent. Information is evolving. Clinical teams need frequent interaction to assimilate the latest reliable information.

2. Strategy 2 Adapt existing tools to enable more efficient collection of critical information compared with developing new tools. Clinical tools help to organize, align, and structure critical information. They guide discussions with patients and providers about testing and treatment and allow the documentation of these communications.

3. Strategy 3 Joint training, using a shared language. Join training for maternal and pediatric providers fosters shared language around critical information. Such communication is best based on physiology as the basis of communication (the $\mathrm{SaO} 2$ is now $88 \%$, Blood Pressures on dopamine have a mean value of $45 \mathrm{~mm} \mathrm{Hg}$ ). Communication needs to be objective, articulate, and succinct, with ongoing collaboration that identifies, interprets and translates words into actions. The CDC, AAP, ACOG, and other maternal and pediatric partners have developed and promoted clinical education packages that increase opportunities for clinicians to build a shared framework to promote communication across specialization areas. Healthcare professions seem to have a perverse joy in their use of specialty jargon as a method to show camaraderie but also to show others' lack of understanding.

4. Strategy 4. Use plain language. Plain-language explanations of laboratory or other results coupled with actionable and defined next steps for provider to provider and provider to patient communications facilitate the collection of information 
and improve interdisciplinary understanding of critical data.

Leeb et al. also address creating information transfer pathways, standardized protocols and procedures, and clear handoffs that include verbal and written components that capture the quantity and detail of shared information (23). Triage and transfer lists are helpful for caregivers, unit managers, and administrators in sending and receiving facilities. In disaster situations, there are challenges to linking medical records across systems of care and patients. An incident command unit may be unfamiliar with the requirements for medical documentation that patients may need to carry with them and to align incentives to support care coordination, especially for pregnant and delivered woman and their babies. Lactation support is essential and metabolic screening, upper and lower extremity blood pressure, and saturation monitoring may be deferred. Hearing screenings all need to be completed, but a patient flow may require that these are done later with more resources. Intentional redundancy reduces communication gaps.

The American College of Obstetrics and Gynecology codified their recommendations in Committee Opinion 726 in 2017 for disasterpreparedness-for-obstetricians-and facilities-providing maternity care (24)

Their specific recommendations include:

- $\quad$ Appoint an obstetrician to direct disaster planning for maternity services, including a pediatrician and maternity and pediatric nursing involvement

- Consider regional patterns of obstetric care provision and disaster scenarios

- Consider obstetric and neonatal needs with high obstetric patient surge

- Establish policies for visitation and lactation that balance infection control concerns with patient and family desires for involvement in the birthing process

- $\quad$ Foster functional working relationships with local and regional critical care clinicians.

- Have a working algorithm for ethical resource allocation that considers obstetric and pediatric-specific needs when demands exceed supply.

- $\quad$ Develop a surge capacity plan realizing the challenges that pregnancy poses to control patient volume

- $\quad$ Consider temporary alterations to usual standards of obstetric care and mechanisms to optimize services with limited resources such as early hospital discharge after delivery, enhanced telephone and telemedicine triage, attention to documentation requirements, and rapid credentialing of health care providers to enable the delivery of obstetric care in the event of workforce limitations.

Disaster action plans are more than an administrative task and testing the feasibility of these plans through mock drills incorporating local and statewide emergency management agencies. It is also essential to find pitfalls or unanticipated difficulties in the execution of disaster responsiveness plans.

\section{Evaluating the Pregnant Patient:}

At the onset of a disaster, the initiation of plans for obstetric and newborn evacuation or "shelter in place" response, and the as- sessment of the available medical personnel, licensed midwives, anesthesia, interpreters, and lactation support, as well as operational resources like delivery kits, medications, and the capacity to maintain a clean environment are essential. Access to existing medical records for obstetric patients, including information of comorbidities, prior laboratory results, and birth plans, may not be fully accessible for pregnant women, those in labor, or who have recently delivered or their newborn. To the degree possible in evacuation centers, a safe and physically protected location should be used to conduct the obstetric interview and physical examination. Either a separate tent or a partitioned-off space will protect women's modesty and potentially improve trust and reduce anxieties. Some cultures eschew the presence of male healthcare providers, and males may not be able to examine women; thus, having a female health worker from the community may help acceptance of male providers to female patients. A complete history for the pregnant woman is not dissimilar to that of the non-pregnant female, but getting information of the woman is crucial for initiation of care:

\section{"Some cultures eschew the presence of male healthcare providers, and males may not be able to examine women; thus, having a female health worker from the community may help acceptance of male providers to female patients."}

\section{Current complaint}

2. Last Menstrual history or when was pregnancy confirmed by a pregnancy test

3. Pregnancy-related symptoms including amenorrhea, breast enlargement and tenderness, nausea and vomiting, increased urination, and fatigue.

4. General medical history for baseline medical conditions, particularly those that could be life-threatening if untreated to the pregnant women (hypertension, diabetes, kidney disease, anemia, maternal infections, seizure disorders, and psychiatric or behavioral disorders

\section{Surgical history}

6. Current Medications and Allergies

7. Vaccination history (including Tetanus, COVID-19, DTaP, Influenza)

8. Obstetric history including gravidity, parity, routes of delivery, premature deliveries, miscarriages, ectopic pregnancies, and prior labor complications (e.g., Uterine rupture, placental abruption, and hemorrhage).

9. Social History including significant other, living conditions, employment, food, and water security, domestic violence, and safety of her surroundings.

A focused physical examination is performed, including vital signs and disaster-related trauma-bruising, lacerations, smoke inha- 
lation, fever, tachycardia, tachypnea, hypo or hypertension, or diminished oxygen saturation must take priority. Uterine size estimation and assessment of fetal heart tones ideally with a Doppler that should be audible by 10-12 weeks (doppler) or 20 weeks using a Pinard stethoscope. Ideally, at an evacuation site or first level of triage, continuous fetal monitoring via cardiotocography is performed on pregnant trauma patients to determine fetal viability. The purpose is overall to assess maternal and fetal well-being or distress and uterine contractions over time. Laboratory testing capabilities may be limited, but most EMS responders can perform point of care testing using an iSTAT device or similar device to determine glucose, Hemoglobin, electrolytes, creatine, blood urea nitrogen, rapid HIV, and COVID-19 testing. At evaluation centers, fetal ultrasound may not be available, and priority evacuation to a $\mathrm{BEmONC}$ or if experiencing premature labor with rupture of members to a CEmONC should be a high priority.

Trauma care for the pregnant patient is beyond this review; however, women with trauma require medical stabilization with a simultaneous focus on fetal well being can often be initiated while the mother is being treated, including placement in the left lat- eral uterine displacement, volume replacement, and supplemental oxygen if available (21). Management of the first and second stages of labor should proceed. However, adaptations to limited resources in less than ordinary environments with cultural sensitivity should acknowledge limited resources with a focus to maintain the mother's safety (21). Post-partum hemorrhage can be immediate or delayed. Immediate post-partum hemorrhage can be caused by an incomplete contracted uterus, vaginal or cervical lacerations, retained placental fragments, or less commonly, uterine rupture, or a combination of these. A non-contracted uterus is treated with fundal massage, and cervical and vaginal lacerations can be repaired with sutures but require adequate lighting for visualization. The placenta is examined to confirm its intactness, and in cases of uterine atony, uterotonic medications and aggressive bimanual massage are initiated, followed by medications to encourage uterine contraction $(25,26)$ ). Efforts must be focused on skin-to-skin maternal-infant dyad care both for the well-being of both mother and baby to provide for breast milk, warmth, and maternal bonding with her newborn.

Equipment and Supplies:

Table I Supply Kits for Use in Perinatal Disaster:

\begin{tabular}{|c|c|c|}
\hline NICU Specific & PICU Specific & OB Specific \\
\hline $\begin{array}{ll}\text { - } & \text { Infant Med Sled } \\
\text { EPOC (Electronic } \\
\text { Point of Care Testing) } \\
\text { T-Piece Resuscitator } \\
\text { Vent } \\
\text { - Chemical warming } \\
\text { mattress } \\
\text { - } \\
\text { Neowrap } \\
\text { Specialized evacuation } \\
\text { equipment for premmies } \\
\text { NICU transport bags } \\
\text { *includes supplies } \\
\text { needed for short term } \\
\text { use } \\
\text { Unit code carts } \\
\text { (removable drawers) } \\
\text { Bedside patient } \\
\text { disaster kits(see NICU } \\
\text { equipment } \\
\text { Section) } \\
\text { Transport Ventilator, } \\
\text { Monitors,Suction, Bed }\end{array}$ & $\begin{array}{ll}\text { - } & \text { Stryker Chair } \\
\text { - } & \text { EPOC (Electronic } \\
\text { Point of Care Testing) } \\
\text { - Portable Pulse } \\
\text { Oximetry } \\
\text { - PICU transport bags } \\
\text { *includes supplies and } \\
\text { medications needed for } \\
\text { short term use } \\
\text { - Unit code carts } \\
\text { (removable drawers) } \\
\text { - Patient Disaster supply } \\
\text { Backpack (see PICU } \\
\text { Supply section) } \\
\text { Transport Ventilator, } \\
\text { Monitors, Suction }\end{array}$ & $\begin{array}{ll}\text { - } & \text { Birthing kits } \\
\text { - } & \text { Eopplers } \\
\text { *off unit delivery } \\
\text { supplies } \\
\text { Postpartum Transport } \\
\text { bags(See OB } \\
\text { Equipment Section) } \\
\text { *includes general } \\
\text { supplies } \\
\text { for mother and baby } \\
\text { Unit code carts } \\
\text { (removable drawers) }\end{array}$ \\
\hline
\end{tabular}


A large influx of patients can quickly deplete a hospital's resources. In the same manner, an evacuation of patients to another facility can also consume resources. Thus, hospitals and individual units must prepare before a disaster strikes. The availability of appropriate and adequate supplies is vital for timely emergency response efforts, so the unit's disaster team should compile a list of essential supplies required to provide routine assessments, respiratory care, nutritional support, and thermoregulation for 12-24 hours in the event of an emergency. However, regulatory agencies require hospitals to have a 96- hour cache of food and supplies or a documented contingency plan on how the hospital will receive these if they do not - keeping equipment powered fully and using emergency power outlets when available and fully charged batteries for backup. Recent disasters such as Hurricane Ida caused power outages, and generators were flooded by hurricane Ida. Thus even redundant power systems may fail and result in hospital evacuation.

"Recent disasters such as Hurricane Ida caused power outages, and generators were flooded by hurricane Ida. Thus even redundant power systems may fail and result in hospital evacuation."

- $\quad$ Units should consider the purchase of backup batteries for equipment and a method to assure charging capabilities and testing based on recommendations of the manufacturer or hospital-based Biomedical/Clinical Engineering and recent advancements in solar power generators that may be ideal in many emergencies.

- Essential equipment for NICU evacuation includes, but are not limited to, the following:

- $\quad$ Portable cardio-respiratory monitors

- $\quad$ Pulse oximetry monitors

- $\quad$ Point of Care Testing (i.e., EPOC, AccuCheck)

- $\quad$ Syringe pumps/IV

- $\quad$ Drug box/Transport bag/Organized supply kit, i.e., Kangaroo Board

- $\quad$ Airway kit and supplies

- $\mathrm{O}_{2}$ source and supplies

- $\quad$ Portable Suction Equipment

- $\quad$ Transport Ventilators, if available

- $\quad$ Consider taking a Crash Cart/Transport Bags if going to a non-clinical Staging Area on a horizontal move

- $\quad$ Consider additional hospital locations of Crash Carts that could easily be moved to the designated Staging Area (i.e., extra NICU Crash Cart maintained in an Outpatient O.B. setting or Emergency Code carts.

\section{Evacuation Equipment}

Recognize that there are multiple types of evacuation equipment explicitly made for specialty populations (i.e., Stryker Chair, Med Sled, neonatal pocket vest). Neonatal evacuation "packages" with sleds to rapidly move multiple infants and supplies are described by Ma et al. (27).

Mobile disaster kits have been developed, from individualized backpacks to unit-specific portable dollies on wheels with equipment, drugs, and other supplies. Patient and supply sleds have been designed that facilitate both vertical and horizontal movement of patients and supplies.

Purchase and direct drills with evacuation supplies and equipment so that staff become familiar with their use.

\section{Maternal-Infant Dyad and support for Infant feeding during disasters}

Infants are particularly vulnerable to illness and death during disasters. Establishing and implementing an infant emergency feeding plan is vital in providing care to families during a disaster. The WHO recommends that infants be exclusively breastfed for the first six months of life; this is also recommended by the American Academy of Pediatrics and the Women Infants and Children (WIC) programs (27). During disasters, breastfed infants are at a decreased risk for dehydration, diarrheal illnesses, and other infections, including respiratory illness, compared to formula-fed infants. In 2018, the World Health Assembly resolution 71.9 urged members to "take all necessary measures to ensure evidencebased and appropriate infant and young child feeding during emergencies, including through preparedness plans, capacitybuilding of personnel working in emergency situations, and coordination of intersectional operations." One recent study evaluating the feeding practices during the mass evacuation during the Fort McMurry wildfire showed a significantly decreased rate in exclusive breastfeeding following the disaster with an odds ratio of $1.96 \mathrm{x}$ higher breastfeeding prevalence before the evacuation (28). Some of the barriers to maintaining breastfeeding during disasters include concerns about adequate space and privacy for breastfeeding mothers and decreased access to support services such as lactation specialists and people knowledgeable in the benefits of breastfeeding.

Additional challenges are posed when the non-targeted distribution of formula occurs during disaster relief efforts. In a publication reviewing the recent literature on barriers and challenges in infant feeding in disasters in middle and high-income countries, they found that the WHO International Code of Marketing and Breastmilk substitutes was violated in all cases. When the formula is distributed without adequate education about proper mixing, access to clean water, and proper space for preparation and cleaning of bottles, there is a risk of creating worse health outcomes in infants. During disasters, mothers may express concerns about decreased milk supply or concern for inadequate nutrition in their milk, leading to mothers incorrectly thinking that formula will be a superior source of nutrition over breastfeeding. Ideally, the formula should only be distributed to mothers who are not breastfeeding. In addition to adequate space and privacy for breastfeeding, consideration for milk storage and an area for pumping should be offered at evacuation centers, with privacy, if possible.

Some mothers may not have access to their breast pump during an evacuation. Having electric pumps, hand pumps, pumping kits, and an area for milk storage can help support mothers who 
Triage for Resource Allocation for inpatients from TRAIN tool (adapted from Lin et al.)

\begin{tabular}{|l|l|l|l|l|l|}
\hline Transport & Car & BLS & ALS & CCS & Specialty \\
\hline Life-support & Stable & Stable & Minimal & Maximal \\
\hline Mobility & Car/Car & Wheelchair/ Seat & $\begin{array}{l}\text { Wheelchair/ } \\
\text { stretcher }\end{array}$ & $\begin{array}{l}\text { Transport rig/ } \\
\text { stretcher }\end{array}$ & $\begin{array}{l}\text { Incubator/ Immo- } \\
\text { bile }\end{array}$ \\
\hline Nutrition & All P.O./ Enteral & $\begin{array}{l}\text { Intermittent/ En- } \\
\text { teral/Partial P.N }\end{array}$ & Continuous & TPN dependent & \\
\hline Pharmacy & P.O. meds & Intermittent IV & IV fluids & IV Drips X1 & IV drips X2 \\
\hline P.O.: taken orally, TPN: total parenteral nutrition, IV: intravenous medications.
\end{tabular}

are exclusively pumping, experiencing a temporary decrease in milk supply, or trying to maintain their milk supply due to separation from their infant. Re-lactation can be considered for recently breastfed infants if there is inadequate access to safe water and formula.

Supplies to consider for Infants and breastfeeding mothers:

- $\quad$ Clean source of water for mixing formula and for drinking

- $\quad$ Disposable breast pads

- $\quad$ Disposable wipes for cleaning pump parts and bottles
- $\quad$ Bottles and bottle nipples

- $\quad$ Nipple shields and nipple cream

- $\quad$ Nursing covers for privacy

- $\quad$ Milk storage containers

- $\quad$ Pump supplies including USB adapter/batteries

While in the U.S., bottled water is usually available for mixing with infant formulas, in the developing world, access to clean water may be less certain. Thus, formula mixed with contaminated water poses a significant health hazard to infants.

\section{Parent Evacuation Preparedness Checklist for Parents: (Table II)}

\begin{tabular}{|l|l}
\hline Emergency Phones Numbers & Parents should have a written list of emergency contacts, not just an electronic
\end{tabular} list on a smartphone: Child's doctor, pharmacy, medical supply Vendor, utility services, and I EMS numbers all on a wallet-sized card.

Emergency Health Information form

Parent completes form documenting the child's list of diagnoses and medications and doses, local pharmacy, dosage, allergies, and equipment settings.

Three or more days of Supplies /Nutrition Parent has a separate 3-day supply of all medications, materials needed for medical equipment (including extra tracheostomy of gastrostomy tubes) and special nutrition) as needed.

Design a "Go Bag"

Parent has a prepacked bag or another container with necessary supplies, including medications that require refrigeration.

Knowledge and equipment available to use Backup batteries generators and power sources

\section{Evacuation plans}

Awareness of Recharging sites

Register with Utility Companies 2019) (32)
Parent has secured power back up for durable medical equipment such as ventilators, nebulizers, infusion pumps, oxygen concentrators Parents are advised to purchase power or solar generators with sufficient voltage and amperage to provide power for equipment

Parents have an established evacuation plan with family, friends, or a designated evacuation site.

Parents are aware of public buildings that allow emergency recharging Sites: police stations, evacuation centers, fire stations, or public library

Parents should register their household and specific equipment with the Utility company providing electricity, water, and natural gas or propane.

(adapted from Gillen, J, Morris M, Preparing Families of Technology-Dependent Children for Emergencies. Hosp Pediatr 


\section{NICU Evacuation and the Neonatologist}

Whether a disaster is predictable, imminent, or has just occurred. Neonatologists, together with essential nurses, respiratory therapists, pharmacists, and clerical support, activate a hopefully predetermined plan of evacuation to safely move infants from the NICU environment to a safer environment while maintaining care duties. When possible, delegating one Neonatologist to communicate with "incident command" while letting others attend to the needs of critically ill infants offers a more seamless response than each Neonatologist communicating through the institutional "chain of command." Getting assistance from associate Neonatologists, pediatricians, and others allows for rapid triage and determination of severity. As reported by others, this workflow supports the care of infants who may be safely and rapidly transported to an evacuation site or for those that require transport with ongoing ventilatory support, vasopressors, and fluid drips using infusion pumps, along with other scheduled medications. A tool kit was developed that guides NICU leadership in developing a comprehensive disaster response plan that complies with the Joint Commission Standards and is based on community best practice models (29). This tool kit discusses "command center" functions and critical elements for an NICU response to disasters(30). Ma and coworkers (31) have described the TRAIN tool (developed by Lin and associates) as a practical way for NICU leaders to categorize the infants under their care daily, should evacuation be necessary (32). Their TRAIN tool assigns ambulance asset needs but not NICU level. According to the need for life support, the transport assignments are categorized as stable (amenable to be done by private vehicle), basic life support, advanced life support, critical care, and specialized transport. Transport support includes mobility issues such as stretchers, incubators, or equipment for highly complex immobile infants and intravenous nutritional and pharmacy support.

\section{"Their TRAIN tool assigns ambulance asset needs but not NICU level. According to the need for life support, the transport assignments are categorized as stable (amenable to be done by private vehicle), basic life support, advanced life support, critical care, and specialized transport. Transport support includes mobility issues such as stretchers, incubators, or equipment for highly complex immobile infants and intravenous nutritional and pharmacy support."}

Depending on resource limitations such as emergency power, access to water, and the availability of needed supplies within the medical center, as many as $80 \%$ of infants can be horizontally transferred to an accepting facility unaffected by the disaster. In disasters, the Neonatologist treats the incident, members of the
NICU staff, the babies under her/his care, and potentially parents at the bedside. Emphasis must focus on maintaining a thermoneutral zone for infants, avoiding smoke, dust, other contaminants, and potential vibrations that may affect gas supply and vacuum lines. As emphasized previously, the primary focus is keeping all babies alive for the moment and determining with staff who can be immediately moved or if a "shelter in place" position is necessary. An infant on ECMO may be very difficult to transport. An infant returning from gastroschisis repair with a silo in place may be challenging, especially with various vasoactive drugs infusing. Coordination requires collaborative action by Neonatologists and Pediatric Surgeons. Staff may need to gather needed supplies, medications, and respiratory therapists may place infants on highflow nasal cannula rather than CPAP to facilitate a move. Ventilated infants may need backup batteries to move infants, providing radiant warmer and ventilator support simultaneously. Infants on TPN may tolerate an interval when IV fluids can be changed to $\mathrm{D} 10 \mathrm{~W}$ or $\mathrm{D} 12.5 \mathrm{~W}$ to sustain glucose delivery and calories until the pharmacy supplies a new TPN preparation. Having evacuation medication and procedure kits already prepared and available within minutes is essential for time-sensitive evacuation, whether vertical or horizontal, because power outages are frequent (as in Hurricane Ida). Multiple infants may be moved using sleds designed to move four infants at a time down a stairwell; staff may also wear "Infant aprons" designed to transport late preterm infants or term infants, usually four at a time. The staff can wear these to move infants to a safe evacuation site rapidly.

More critically ill infants such as those on the ventilator often require infant transport units as customarily used for infant transports between centers. Transport ventilator, E cylinders for air and oxygen, and batteries usually provide a safe environment to move most infants requiring assisted ventilation. It is helpful and essential to have transport agreements among NICUs within a region or state to transport seamlessly. When entire regions are affected, statewide affiliations or interstate transport agreements may be required; however, it is already occurring when critically ill infants are moved across stateliness to facilitate their care.

For all infant care levels, redundant systems for electrical power, medical gases, wall suction, information technology, and drinkable water are advised. Although hospital-wide generators constitute a significant source of electrical power, NICU-specific power generators may be needed, as recently demonstrated at New Orleans Children's Hospital, where Hurricane Ida flooded hospital generators. Recent wildland fires resulted in several days of poor air quality with air quality indices $>150$ resulting in staff and probably infant discomfort breathing ambient smoke-filled air. Adequate ventilation systems with air filters and cycled air exchange will maintain ambient conditions potentially less injurious to the airways of infants who are breathing ambient air or an air/oxygen mixture.

\section{Technology Dependent infants and Children, family pre- paredness:}

Care for technology-dependent, often fragile infants and children is a challenge for hospital evacuations and parents at home if a disaster occurs. Children who have ongoing medical needs and mobility challenges, those who require continuous power sources, oxygen at variable flow rates, and specialized nutrition, whether through continuous feeding tubes or gastrostomies, place additional responsibilities on their parents and potential rescuers in 
a disaster. Families and other caregivers must understand their infant or child's ongoing needs and the unique risks under those circumstances.

The disaster planning information needs of such families and the parent's communication preferences have been understudied, as have the forms of education and material support for an effective disaster-prepared family plan. Gillen and Morris (32) have stressed the importance of creating a disaster plan for parents as a part of discharge planning from pediatric intensive care units or neonatal intensive care units with follow-up over six months to determine information retention and actual preparedness by parents (33). Others have measured the impact of having a disaster preparedness supply starter kit for parents and report that inexpensive educational disaster supply starter kits may increase parental preparedness, as noted in the table. (34)

\section{"Depending on the locale of residence, it is critical for parents of technology- dependent to notify local Emergency Management offices (usually at the county level) regarding their infant's or children's particular needs and requirements for technology assistance."}

Depending on the locale of residence, it is critical for parents of technology-dependent to notify local Emergency Management offices (usually at the county level) regarding their infant's or children's particular needs and requirements for technology assistance. Addresses, telephone numbers, cross streets, or other identifiers should be given to local police and Emergency Medical Services offices. In areas where power outages occur, whether because of weather, fire, or flooding having a home generator is ideal as an alternative electric source or battery backup, and having at least a 3-day supply of oxygen, medications for aerosolization, and nutrition is a minimum need. When evacuation must occur rapidly, having these items in a central location ready to move at a moment's notice is critical. Experiences during Hurricane Katrina, Superstorm Sandy, and recent wildfires illustrate that a parent's preparation for their child's ongoing special health care needs is obligatory. Evacuation centers often lack the supplies unique to each child's special needs or individual technological requirements. Neonatologists and Pediatricians share duties with discharge planners, nursing and respiratory care practitioners. They all have critical roles in carefully listing anticipated needs for a child with special needs and reinforcing the need for parents to be prepared in the event of a disaster. Follow-up by home-health support workers to ensure that parents have adhered to these recommendations is also critical. Redundant communication to support adherence is vital to ensure compliance.

In California, there is a county registration for individuals with special needs. For example, San Luis Obispo County, Ca, provides a registration form (as illustrated) for a countywide registry for individuals with disabilities and special needs. While these registries are voluntary, they allow county emergency services managers to prioritize their response within the county to those with the high- est needs in case a disaster occurs. A similar county registration has been proposed for Oregon and is under consideration by the Oregon Health Authority.

\section{Demobilization, Unit Recovery, and After-Action Debriefing and Support:}

Demobilization refers to activities that focus on disengaging resources and personnel after the objectives of the disaster response have been met and operations return to normal functioning. In contrast, recovery efforts include long-term activities such as rehabilitating personnel, repairing or replacing equipment, and restocking resources.

Several steps should be followed in the emergency operations plan and considered a priority in the recovery after a disaster to facilitate unit recovery and systems improvement. These steps should include:

1. Uniting families with patients

2. Inventories or equipment and supplies

3. Evaluating of equipment function or failure to do so

4. Reordering and replacement of supplies

5. Assessing the usefulness of communication during the disaster (written, electronic medical records, radio/telephone contacts)

6. Returning to normal staffing patterns as soon as possible

7. Conducting debriefing for all staff involved in the incident

8. Completion of "After-Action reports" to be sent to the Emergency Management Program Director

9. Monitoring of mental health of staff, patients, and visitors and providing emotional support for all.

\section{Discussion:}

Disaster preparedness and response requires both individual and team training and readiness. Situational awareness, analysis, and response by those "on the ground" are essential elements of effective disaster response. Successful disaster medical personnel share many of the following attributes (25):

\section{Capability to work as a team in hardship positions}

2. Ability to multitask and be multifunctional

3. Ability to integrate well into a resource-limited and regimented environment

4. Capability of making complex and difficult decisions

5. Ability to work cooperatively within the hierarchical structure of the disaster response team

Health care facilities should identify workers with these characteristics and organize a disaster-prepared team. They should develop plans and buy the needed equipment. Institutional responsibility and support are paramount in disaster preparedness and delivering health care to mothers, infants, and children during and following an unexpected crisis.

This responsibility also falls on the government to support and ensure the survival and protection of the community it serves.

The Western Regional Alliance for Pediatric Emergency Manage- 
ment (WRAP-EM) has recently received a $\$ 3$ million grant to develop one of two Pediatric Disaster Care Centers of Excellence in the U.S. They are developing a model for improving emergency pediatric disaster response capabilities throughout the Western U.S. and to address weaknesses in emergency communications or access and uncover unknown challenges that could be detrimental during crises. Multiple institutions in Washington, Oregon, California, Nevada, and Arizona representing some 13 million children will gather 60 regional experts and subject matter experts to develop a more highly focused plant to inform the capacity and capabilities on the West Coast in response to disasters affecting pediatric patients. The WARP-EM consortium of some 60 institutions and medical centers will execute a year-long project in phases. They will be conducting a comprehensive review of available resources, developing a pediatric resource library, and conducting a gap analysis to assess the group's existing ability to respond to emergencies. A second phase will focus on delivering products with a measurable impact, such as creating a published guide containing regional pediatric disaster response expertise, developing telemedicine interconnectedness across the Western U.S., establishing a comprehensive pediatric training and educational platforms, and implementing patient tracking, reunifications, and information sharing practices to address interstate operational challenges (30). While this laudable effort may develop a network NICU with a more coordinated response to disasters, it is unclear how the NICUs are chosen to participate and whether they represent NICUs in the most disaster-prone regions in the Western U.S.

"While this laudable effort may develop a network NICU with a more coordinated response to disasters, it is unclear how the NICUs are chosen to participate and whether they represent NICUs in the most disaster-prone regions in the Western U.S."

Manuscripts in this forthcoming series will study disasters affecting NICUs and Neonatologists' responses to various disaster types.

General and President Dwight D. Eisenhower said, "The plan is useless; it's the planning that's important."

\section{References:}

1. Vogus, T, Weick, K, Sutcliffe, Doing No Harm: Enabling, Enacting, and Elaborating a Culture of Safety in Health Care. Acad. Management Perspectives 2010;24(4):60-77.

2. Rasmussen J. Risk Management in a dynamic society. Safety Science, 1997,; 27 (2-2):183-213.

3. CHA Hospital Preparedness Program (https://www.calhospitalprepare.org/cha-tools, accessed 8/42.

4. Kaiser Permanente Human Hazards: kp_hava_template-2014.x/s accessed 8/4/21.

5. Hanenburg, T, Kitchen S, Fitzgerald S. How Kaiser Permanente Prepares for Disasters. Harvard Business Review, December 26, 2019: (https://hbr.org/2019/12/how-kaiserpermanente -prepares-fir-disasters. Accessed 8/4/21
6. Phillips, $P$, Niedergesaess, $Y$, Powers $R$, et al. Disaster preparedness: Emergency Planning in the NICU. Neonatal Network 2012, 31:z

7. Carbine D. CAN Toolkit for NICU Disaster Preparedness. Cdphready.org/neonatal=disaster=preparedness=toolkit (accessed 8/1/21)

8. California Association of Neonatologists (CAN) Neonatal Disaster Preparedness Toolkit. 2015 cpqcc.org/content/ can-neonatal-disaster-preparedness-toolkit. 8/1/2021)

9. Eskandar-Afshari, F, Carbine D, Cohen, R, Cui et al. California NICU disaster preparedness. J. Pernatol. 2020; 40:12621266.

10. Eskandar, A, Lee H, Carbine, Chu, X. California NICU Disaster Preparedness. Pediatrics, 2020; 141:319-321 (Abstract)

11. Barfield, W, Krug SE and Committee of Fetus and Newborn, Disaster Preparedness Advisory Council, Pediatrics 2017; 132(5), May 2017 e20170607.

12. Krug, S, Chung, S, Fagbuly, D, Fisher M, Needle, S., Schonfeld, D. Pediatric Preparedness Resource Kit, https://www. aap. Org/en-us/advocacy-and-policy/aap-health-initiatives/ children-and-disasters/documents/ped. Accessed 8/1/21).

13. International Federation of Red Cross and Red Crescent Societies. What is a disaster? Available at www.ifrc.org/en/ wha-we-do/disaster-managment/about-disastrs/what-is-adisaster/ accessed 8/1/2021).

14. Zotti, ME, Williams, AM, Robertson $M$ et al. Post-disaster reproductive health outcomes. Matern. Child Health J 2013; 17: 783.

15. Chi PC, Urdal H, Umeora OU et al. Improving maternal, newborn and women's reproductive health in crisis settings. Cochrane Database Syst Rev 2015; 8:1.

16. Callaghan WM, Rasmussen SA, Jamieson DJ et al. Health concerns of women and infants in times of natural disasters: lessons learned from Hurricane Katrina Matern Child Health J. 2007; 11:307.

17. Harville $E$, Xiong $X$, Beukens $P$. Disasters and perinatal health: a systematic review. Obstetr Gynecol Surv. 2010;65: 713.

18. Van den Akker T, de Vroome S, Mwagomba B. et al. Peripartum infections and associated maternal mortality in rural Malawi. Obstet Gynecol 2011; 118: 266.

19. Alderman K, Turner LR, Tong S, Floods and human health.: a systematic review. Environ Int 2012; 47:37.

20. Xiong, Harville, EW, Mattison DR., et al. Exposures to Hurricane Katrina post-traumatic stress disorder and birth outcomes. Am J Med Sci 2008; 336:111.

21. Tong VT, Zotti ME, Hsia J. Impact of the Red River catastrophic flood on women giving birth in North Dakota. 19942000. Matern Child Health J 2011:15:281.

22. Medecins Sans Frontiers (Doctors without Borders). Essential obstetric and newborn care. A practical guide for midwives, doctors with obstetrics training and health care personnel who deal with obstetric emergencies. 2015. http:// refbooks.msf.org/msf docs/en/obstetrics/obstetrics en pdf. Accessed 8/1/2012).

23. Leeb, RT, Cree, RA, Aird L, et al. A Framework for Coordination between Obstetric and Pediatric Providers in Public Health Emergencies: Lessons Learned from the Zika Outbreak in the United States, 2015 to 2017. Am J Perinatol 2020, 37(10): 982-990. 
24. ACOG Opinion Statement 726 https://www.acog.org/clinical/ clinical-guidance/committee-opinion/articles/2017/12/hospital -disaster-preparedness-for-obstetricians-and facilitiesproviding maternity care. Accessed 8/1/2021.

25. Huls Ck, Detiefs C. Trauma in pregnancy. Semin Perinatol 2018:42:13.

26. Medecins Sans Frontiers (Doctors Without Borders). Essential obstetric and newborn care. A practical guide for midwives, doctors with obstetrics training, and health care personnel who deal with obstetric emergencies. 2015. http:// refooks.msf.org/msf docs/en/obstetris/obstetricsEen. Pdf (accessed 8/2/2021)

27. WIC Breastfeeding Support (n.d.) Breastfeeding during disasters. Retrieved August 24, 2021. From https://wicbreastfeeding.fsn.usda.gov/breastfeeding-during-disasters

28. DeYoung, Sarah et al. The Effect of Mass evacuation on Infant Feeding: The Case of the 2016 Fort McMurray Wildfire. Maternal and Child Health Journal (2018) 22:1826-1833.

29. Carbine D, Cohen R, Hopper, A California Association of Neonatologists (CAN): Neonatal Disaster Preparedness Toolkit; California Perinatal Quality Care Collaborative (CPQCC): Stanford, CA, USA, 2015; 3-47.

30. New York City Pediatric Disaster Coalition; New York City Department of Health and Mental Hygiene. Neonatal Intensive Care Unit Surge and evacuation Plan Template; NYC Health. New Your, NY, USA, 2018;6.

31. Ma A, Cohen R, Lee $H$. Learning from Wildfire Disaster EXperience in California NICUs. Children. 2020; 7, 155.

32. Lin A, Taylor, $K$, Cohen R Triage by Resource Allocation for Inpatients: A Novel disaster Triage Tool for Hospitalized Pediatric Patients. Disaster Med Public Health Prep 2018, 12: 692.

33. Gillen J, Morris M. Preparing Families of Technology-Dependent Children for Emergencies. Hosp. Pediatr. 2019; 11:874.

34. Bagwell, H, Liggin, R, Thompson T et al. Disaster Preparedness in Families with Children with Special Health Care Needs. Clin Pediatr 2016; 112: 1036.

35. King RV, Larkin G, Fowler R. et al. Characteristics of Effective Disaster Responders and Leaders: A Survey of Disaster Medical Practitioners. Disaster Med Public Health Prep 2016: 10: 720.

Conflict of Interest: The authors have indicated they have no potential conflict of interest relevant to this article to disclose.

NT
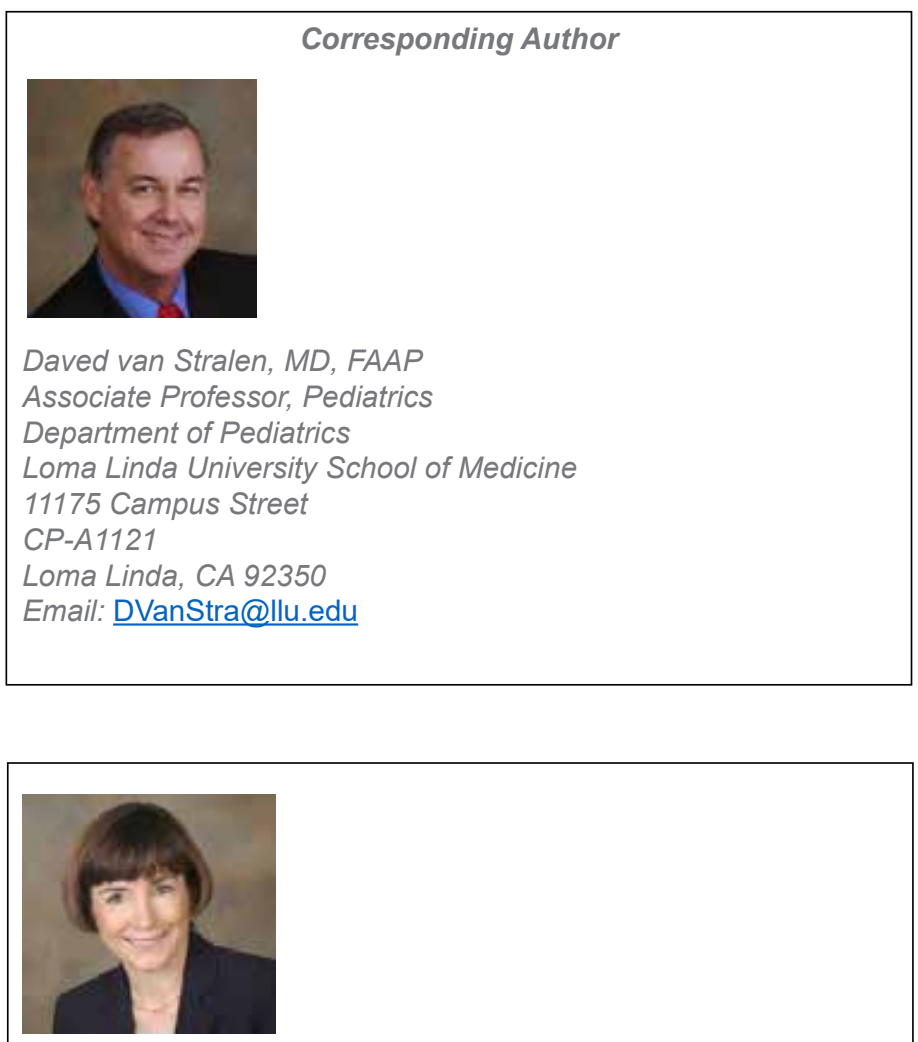

Elba Fayard, MD

Professor of Pediatrics

Division Chair

Division of Neonatology-Perinatal Medicine

Loma Linda University Children's Hospital

Loma Linda, CA

Email: Efayard@/lu.edu

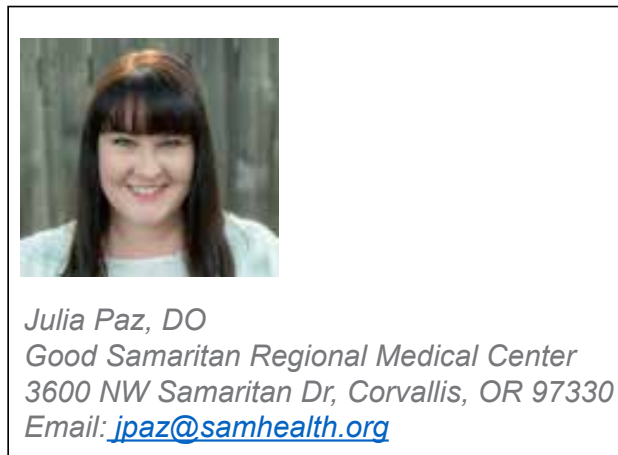

Email:jpaz@samhealth.org

NEONATOLOGY TODAY is interested in publishing manuscripts from Neonatologists, Fellows, NNPs and those involved in caring for neonates on case studies, research results, hospital news, meeting announcements, and other pertinent topics. Please submit your manuscript to: LomaLindaPublishingCompany@gmail.com 